\title{
Investigation of tooth transposition in a non-syndromic Turkish anatolian population: Characteristic features and associated dental anomalies
}

\author{
Mevlut Celikoglu ${ }^{1}$, Ozkan Miloglu ${ }^{2}$, Ozkan Oztek
}

\footnotetext{
${ }^{1}$ Research Assistant, Department of Orthodontics, Faculty of Dentistry, Ataturk University, Erzurum, Turkey

${ }^{2}$ Assistant Professor, Department of Oral Diagnosis and Radiology, Faculty of Dentistry, Ataturk University, Erzurum, Turkey
}

Correspondence:

Department of Orthodontics

Faculty of Dentistry

Ataturk University

Erzurum, 25240, Turkey

mevlutcelikoglu@hotmail.com

\begin{abstract}
Celikoglu M, Miloglu O, Oztek O. Investigation of tooth transposition in a non-syndromic Turkish anatolian population: Characteristic features and associated dental anomalies. Med Oral Patol Oral Cir Bucal. 2010 Sep 1;15 (5):e716-20.
\end{abstract}

http://www.medicinaoral.com/medoralfree01/v15i5/medoralv15i5p716.pdf

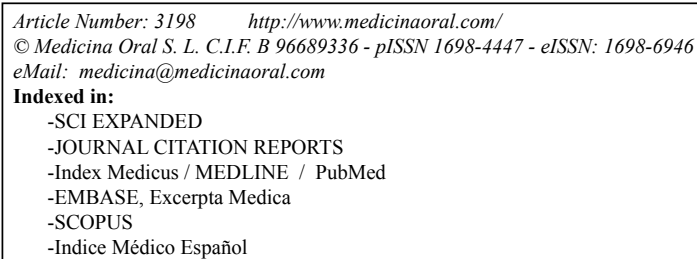

\begin{abstract}
Objective: The aims of this study were to investigate the frequency and characteristics of dental transpositions and to evaluate associated dental anomalies in a large sample of Turkish Anatolian population.

Study design: A retrospective study was performed using panoramic radiographs of 6983 patients (4092 females and 2891 males) ranging in age from 12 to 27 subjected to Faculty of Dentistry at the University of Ataturk (Erzurum, Turkey) between 2005 and 2008. For each patient with tooth transposition we recorded the demographic variables (including age, sex), history of trauma, type, classification, and location of tooth transpositions, and associated dental anomalies. The Pearson chi-squared test was used to determine potential differences in the distribution of tooth transposition when stratified by gender.

Results: Tooth transposition was detected in 19 subjects $(0.27 \%)$, with a $2.2: 1$ female male ratio $(\mathrm{P}=0.38)$. The most commonly observed transposition was maxillary canine-lateral incisor $(60 \%)$. The frequencies of complete and incomplete transpositions were equal (10/10) and it was more common in the left side than in the right side (11/9). Of the 19 subjects, $10.5 \%$ had a peg shaped lateral incisor, $21.1 \%$ one congenitally missing tooth excluding third molar. Supernumerary tooth, impacted teeth excluding third molars, transmigrated tooth, and dilacerations were also observed.

Conclusion: The frequency of tooth transposition was $0.27 \%$ in a Turkish Anatolian population and maxillary canine-lateral incisor was the most frequently observed transposition. Retained primary teeth were the most frequently observed dental anomaly in all types of tooth transposition.
\end{abstract}

Key words: Transposition, prevalence, ectopic eruption. 


\section{Introduction}

Dental transposition is the positional interchange of two adjacent teeth, or the development or eruption of a tooth in a position normally occupied by a non-adjacent tooth (1). It is identified as "complete" when the crowns and the roots of the involved teeth exchange places in the dental arch and "incomplete" when the crowns are transposed, but the roots remain in their normal positions.

Tooth transposition seems to affect almost equally males and females, and it affects the permanent dentition both unilaterally and bilaterally, with a greater frequency of unilateral cases (2). The maxillary canine is probably the tooth most frequently displaced. When displaced in the palatolabial plane, it may become palatally or labially impacted. When displaced mesially or distally, an ectopically erupting canine may become transposed with one of the adjacent teeth (3).

It generally occurs in the maxilla and is associated with other dental anomalies, such as peg-shaped lateral incisors, retained deciduous canine, supernumerary teeth, several rotations and malpositions, dilaceration, hypodontia, and impacted teeth $(1,2,4)$.

Although transposition was defined in the 19th century, its aetiology is still uncertain. Heredity, trauma, syndromes, retained deciduous canine, migration of a tooth during eruption, and transposition of tooth buds at anlage stage were proposed as possible aetiological factors $(3,5)$. Bone pathology, such as cyst formation and odontomas may also cause displacement and transposition of the tooth (6).

Transpositions are classified according to Peck and Peck's classification (7): maxillary canine-first premolar (Mx.C.P1), maxillary canine-lateral incisor (Mx.C.I2), maxillary canine-first molar site (Mx.C to M1), maxillary lateral incisor-central incisor (MxI2.I1), maxillary canine-central incisor site (Mx.C to I1), and mandibular lateral incisor-canine (Mn.I2.C).

The aims of this study were to investigate the frequency and characteristics of dental transpositions and to evaluate associated dental anomalies in a large sample of Turkish Anatolian population.

\section{Material and Methods}

A retrospective study was performed using panoramic radiographs of 6983 patients (4092 females and 2891 males) ranging in age from 12 to 27 (mean age 15.9 years) subjected to Faculty of Dentistry at the University of Ataturk (Erzurum, Turkey) between 2005 and 2008. Selection criteria of the samples included the patients that were not diagnosed with any syndrome or illness involved odontogenesis and dental eruption. The followings were recorded for each subject with tooth transposition: Age, sex, history of trauma, type, classification and location of tooth transpositions, and presence of associated dental anomalies, such as supernumerary teeth, impacted teeth, hypodontia, peg-shaped lateral incisor, retained primary teeth, and transmigrated tooth (Fig. 1, 2, and 3).

All radiographs were reviewed and discussed by the panel in a negatoscope an a7x lens was used. To check for the diagnostic reproducibility of the interreliability of the two investigators, $10 \%$ of the radiographs assigned by them were randomly examined each day for

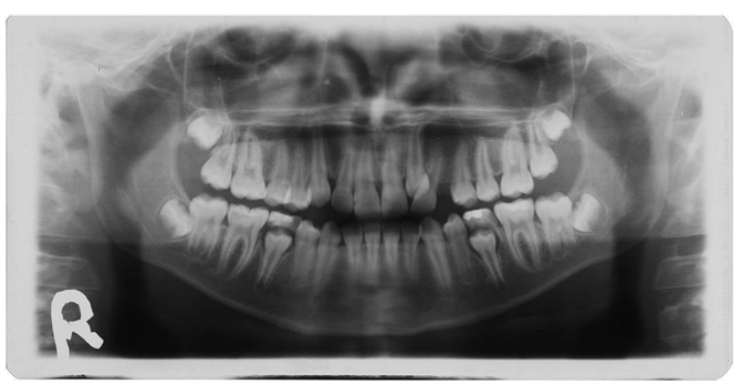

Fig. 1. Complete transposition of the maxillary left permanent canine and lateral incisor.

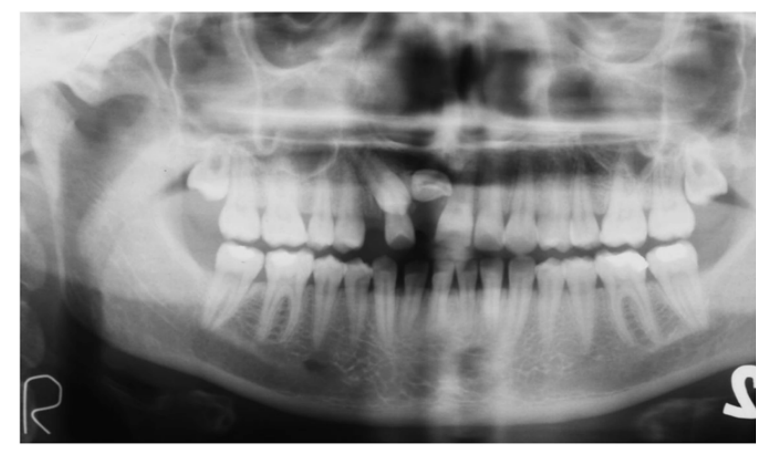

Fig. 2. Incomplete transposition of the maxillary right permanent canine and lateral incisor with associated impacted central incisor.

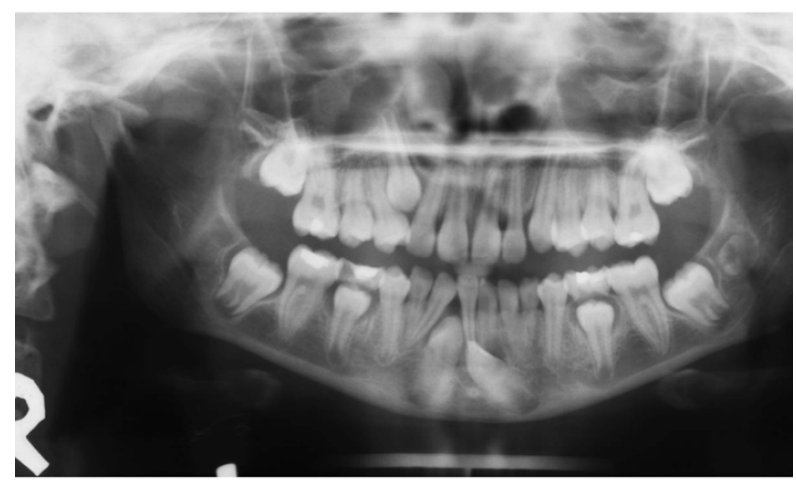

Fig. 3. Complete transposition of the mandibular right permanent lateral incisor and canine with transmigrated left permanent canine. 
three consecutive days. Examination of results using the Wilcoxon matched-pairs signed rank test showed no statistically significant differences between the two observers, indicating diagnostic reproducibility. The Pearson chi-squared test was used to determine potential differences in the distribution of tooth transposition when stratified by gender. A $p$ value of $<0.05$ was considered statistically significant.

\section{Results}

Tooth transposition was detected in 19 subjects $(0.27 \%)$, of which 6 were males and 13 were females with a 2.2:1 female male ratio $(\mathrm{P}=0.38)$ (Table 1).

Frequency and characteristic features of each type of tooth transpositions and associated dental anomalies are shown in (Table 2). All of 19 subjects had transpositions involving the canine tooth. The most commonly observed transposition was Mx.C.I2 (60\%), secondly Mx.C.P1 (30\%), and Mn.I2.C was the least commonly observed transposition (10\%). The majority of the transpositions (18 of 20) were in the maxillary arch and it was more common in the left side than in the right side (11/9). Only one subject had bilateral transposition. The frequencies of complete and incomplete transpositions were equal (10/10). No subject had transpositions in both the maxillary and mandibular arches.
Of the 19 subjects, $57.9 \%$ had retained primary teeth, $10.5 \%$ had a peg shaped lateral incisor, and $21.1 \%$ had one congenitally missing tooth excluding third molars. Supernumerary teeth was observed in $5.3 \%$ of the cases, impacted teeth excluding third molars in $10.5 \%$, transmigrated teeth in $5.3 \%$ and dilacerations in $10.5 \%$. Retained primary teeth were the most frequently observed dental anomaly in all transposition types. Previous trauma to the transposed area was detected in one case.

\section{Discussion}

The frequency of the tooth transposition $(0.27 \%)$, in the present study, is very close to the data reported by Yllmaz et al. (4) and Shapira and Kuftinec (3), who stated that the prevalence of tooth transposition was $0.4 \%$. Some reports $(1,3)$ showed that transpositions were more frequently observed in females while some reported the opposite $(4,8)$. Chattopadhyay and Srinivas (8) explained that higher transposition frequency in females might be due to the higher numbers of females seeking orthodontic treatment. On the other hand, the approximate 2:1 ratio of female to male found in the present study is in agreement with many reports (1-3) that indicate female predominance for tooth transposition. However, the Pearson chi-square test showed no statistical difference $(\mathrm{P}=0.38)$.

Table 1. Distribution of the tooth transposition according to the gender.

\begin{tabular}{|c|c|c|c|c|c|}
\hline Gender & $\mathbf{n}$ & Transposed teeth & Frequency (\%) & P value & Total (\%) \\
\hline Female & 4092 & 13 & 0.32 & 0.38 & $19(0.27)$ \\
\hline Male & 2891 & 6 & 0.21 & & \\
\hline
\end{tabular}

Table 2. Frequency and characteristics of the transposition cases.

\begin{tabular}{|l|c|c|c|c|}
\hline & Mx.C.P1 & Mx.C.I2 & Mn.I2.C & Total \\
\hline No of cases & $6(0.09 \%)$ & $11(0.16 \%)$ & $2(0.03 \%)$ & $19(0.27 \%)$ \\
\hline Male:Female & $2: 4$ & $3: 8$ & $1: 1$ & $6: 13$ \\
\hline Complete:Incomplete & $3: 3$ & $5: 7$ & $2: 0$ & $10: 10$ \\
\hline Left:Right & $3: 3$ & $8: 4$ & $0: 2$ & $11: 9$ \\
\hline Retained teeth & $50 \%(3 / 6)$ & $54.5 \%(6 / 11)$ & $100 \%(2 / 2)$ & $57.9 \%(11 / 19)$ \\
\hline Missing teeth & $33.3 \%(2 / 6)$ & $18.2 \%(2 / 11)$ & - & $21.1 \%(4 / 19)$ \\
\hline Peg laterals & $16.7 \%(1 / 6)$ & $9.1 \%(1 / 11)$ & - & $10.5 \%(2 / 19)$ \\
\hline Impacted teeth & - & $18.2 \%(2 / 11)$ & - & $10.5 \%(2 / 19$ \\
\hline Transmigrated teeth & - & - & $50 \%(1 / 2)$ & $5.3 \%(1 / 19)$ \\
\hline Dilaceration & $16.7 \%(1 / 6)$ & $9.1 \%(1 / 11)$ & - & $10.5 \%(2 / 19)$ \\
\hline Supernumerary teeth & $16.7 \%(1 / 6)$ & - & - & $5.3 \%(1 / 19)$ \\
\hline Trauma & $16.7 \%(1 / 6)$ & - & - & $5.3 \%(1 / 19)$ \\
\hline
\end{tabular}


Tooth transpositions most frequently involve the canine tooth, which is transposed with either the first premolar or the lateral incisor $(1,3,4)$. Transpositions that do not involve canine teeth, such as the central and lateral incisor transpositions, are extremely rare $(1,7)$. Similarly, in the present study, the canine teeth were involved in all cases and the most frequently observed transposition was found to be Mx.C.I2. This value is in agreement with the data reported by Chattopadhyay and Srinivas (8) and Yilmaz et al. (4), who reported a higher frequency of Mx.C.I2 transposition. However, this differs from Plunkett et al. (2) and Shapira and Kuftinec (3) reports of a much greater frequency of Mx.C.P1 transposition. With caution, there seems to be some predominance of the maxilla over the mandible in tooth transposition. The high density of bone in the mandible might prohibit tooth transposition, hence justifying the higher frequency of maxillary occurrence (9). According to our results, maxillary transposition accounted for the majority of the cases $(90 \%)$, which is in agreement with previous studies (1-3). In contrast to the maxilla, mandibular transposition was found to be a less common phenomenon $(10 \%)$, in which all of them involved Mn.I2.C. This result is in agreement with the data reported by Plunkett et al. (2) and Peck and Peck (7), who stated that the Mn.I2.C was the most frequently observed transposition in the mandibular arch.

In some studies $(1,3,8)$ greater frequency of left side occurrence was reported with no satisfactory explanation. Yilmaz et al. (4) found the prevalence of tooth transposition in the left and right sides to be equally affected. On the other hand, our results showed higher frequency of tooth transposition in the left side than in the right side (11/9). However, all of the mandibular transpositions were in the right side. This interesting finding is consistent with the data reported by Yilmaz et al. (4), who stated that all of the mandibular transposition was in the right side.

Unilateral transposition was, as expected, encountered more frequently than bilateral transposition (18 to 1 respectively). This was in agreement with previous reports (1-3).

Agenesis of teeth in the general population has a frequency of 3.5 to $8 \%$ (1). Yilmaz et al. (4) and Chattopadhyay and Srinivas (8) found tooth agenesis in transposition cases to be $33.3 \%$ and $40 \%$, respectively. In the present study, tooth agenesis was observed in $21.1 \%$ of the cases. Peg-shaped lateral incisors occur in approximately $1 \%$ of the general population (10) and supernumerary teeth in 1.05 (11) to $3.2 \%$ (12). Chattopadhyay and Srinivas (8) reported a higher frequency of peg-shaped lateral incisor in the Mx.C.P1 transpositions (67\%) and Mx.C.I2 transpositions (7.1\%). However, Shapira and Kuftinec (3) found that only one subject $(1 / 65)$ had pegshaped lateral incisor. Although it appears that subjects with tooth transpositions do have a higher frequency of other dental anomalies, it is not possible to determine from the present study whether tooth transposition has a genetic aetiology. On the other hand, Baccetti (13) reported that the associations among several other dental anomalies suggest that the conditions may share a common genetic origin.

Deciduous canines have often been found to be over retained in a majority of the transposition cases $(1,3,4,8)$. In agreement with those reports, we found retained deciduous canine teeth in approximately $52 \%$ of maxillary transposition, while $100 \%$ of mandibular transpositions. This result indicated that it might be the main aetiological factor for Mn.I2.C transposition. However, the question of whether the deflection of the permanent canine teeth cause the deciduous canine to be retained or, if the retained deciduous canine causes the tooth transposition remains a matter of pure speculation (3). Trauma to the deciduous teeth has also been suggested as a factor in the aetiology of tooth transposition (14). Although Türkkahraman et al. (15) and Yilmaz et al. (4) suggested that trauma was the main aetiological factor for tooth transposition, in this study it was observed in $5.3 \%$ of the cases. Therefore, trauma might be only possible aetiological factor for tooth transposition.

Finally, the frequency of tooth transposition was $0.27 \%$ in a Turkish Anatolian population and maxillary canine-lateral incisor was the most frequently observed transposition. Retained primary teeth were the most frequently observed dental anomaly in all types of tooth transposition.

\section{References}

1. Peck L, Peck S, Attia Y. Maxillary canine-first premolar transposition, associated dental anomalies and genetic basis. Angle Orthod. 1993;63:99-109.

2. Plunkett DJ, Dysart PS, Kardos TB, Herbison GP. A study of transposed canines in a sample of orthodontic patients. Br J Orthod. 1998;25:203-8.

3. Shapira Y, Kuftinec MM. Maxillary tooth transpositions: characteristic features and accompanying dental anomalies. Am J Orthod Dentofacial Orthop. 2001;119:127-34.

4. Yilmaz HH, Türkkahraman H, Sayin MO. Prevalence of tooth transpositions and associated dental anomalies in a Turkish population. Dentomaxillofac Radiol. 2005;34:32-5.

5. Alves-Pereira D, Berini-Aytés L, Gay-Escoda C. Ellis-van Creveld syndrome. Case report and literature review. Med Oral Patol Oral Cir Bucal. 2009;14:E340-3.

6. Serra-Serra G, Berini-Aytés L, Gay-Escoda C. Erupted odontomas: a report of three cases and review of the literature. Med Oral Patol Oral Cir Bucal. 2009;14:E299-303.

7. Peck S, Peck L. Classification of maxillary tooth transpositions. Am J Orthod Dentofacial Orthop. 1995;107:505-17.

8. Chattopadhyay A, Srinivas K. Transposition of teeth and genetic etiology. Angle Orthod. 1996;66:147-52.

9. Papadopoulos MA, Chatzoudi M, Karagiannis V. Assessment of characteristic features and dental anomalies accompanying tooth transposition: a meta-analysis. Am J Orthod Dentofacial Orthop. 2009;136:308.e1-10.

10. Meskin LH, Gorlin RJ. Agenesis and peg-shaped permanent 
maxillary lateral incisors. J Dent Res. 1963;42:1476-9.

11. Leco Berrocal MI, Martín Morales JF, Martínez González JM.

An observational study of the frequency of supernumerary teeth in a population of 2000 patients. Med Oral Patol Oral Cir Bucal. 2007;12:E134-8.

12. Salcido-García JF, Ledesma-Montes C, Hernández-Flores F, Pérez D, Garcés-Ortíz M. Frequency of supernumerary teeth in Mexican population. Med Oral Patol Oral Cir Bucal. 2004;9:407-9; 403-6.

13. Baccetti T. A controlled study of associated dental anomalies. Angle Orthod. 1998;68:267-74.

14. Dayal PK, Shodhan KH, Dave CJ. Transposition of canine with traumatic etiology. J Indian Dent Assoc. 1983;55:283-5.

15. Türkkahraman H, Sayin MO, Yilmaz HH. Maxillary canine transposition to incisor site: a rare condition. Angle Orthod. 2005;75:284-7. 